\title{
Patients with Mood Disorders Require Higher Doses of Buprenorphine for Management of Opi- oid Use Disorder But Have No Increased Risk of Neonatal Abstinence Syndrome
}

Tiffany Tonismae, $\mathrm{MD}^{1,2,3}$, Misty McDowell, $\mathrm{MD}^{1}$, Loraine Torres, $\mathrm{MD}^{3}$, James E. Slaven, MS, MA ${ }^{4}$, Sara K Quinney, PharmD, $\mathrm{PhD}^{1,5}$, Frank Schubert, MD, MS ${ }^{1}$, Mary Pell Abernathy, $\mathrm{MD}^{1}$

1. Indiana University School of Medicine, Department of Obstetrics and Gynecology, Division of Maternal Fetal Medicine, Indianapolis, Indiana

2. Johns Hopkins All Children's Hospital, Maternal Fetal \& Neonatal Institute, St. Petersburg, Florida

3. Bayfront Health St. Petersburg, Department of Obstetrics and Gynecology, St. Petersburg, Florida

4. Indiana University School of Medicine, Department of Biostatistics \& Health Data Science, Indianapolis, Indiana

5. Indiana University School of Medicine, Division of Clinical Pharmacology, Department of Medicine, Indianapolis, Indiana

\section{Abstract}

Objective: This study compared differences in buprenorphine doses needed to treat opioid use disorder in pregnant women with and without mood disorders and to compare the development of neonatal abstinence syndrome in infants delivered to mothers treated with buprenorphine in patients with history of mood disorders versus those without mood disorder.

Methods: This retrospective cohort study included women with opioid use disorder prescribed buprenorphine who had at least one outpatient visit at with the Indiana University Department of Maternal Fetal Medicine during pregnancy and delivered within the Indiana University Health system. Charts were reviewed for maternal demographics, medical history and medication use, and neonatal outcomes. Cases included those patients with history of mood disorder including depression, anxiety, or post-traumatic stress disorder based on initial appointment intake forms. Starting and maximum doses of buprenorphine during pregnancy were recorded. Outcomes were compared using Student's t-tests and Analysis of Variance models for continuous variables and chi-square tests for categorical variables. All analytic assumptions were verified, with non-parametric tests being performed where necessary.

Results: A total of 266 women were treated with opioids, of which 171 were diagnosed with a mood disorder: 148 depression, 130 anxiety, and 19 post-traumatic stress disorder. Over $40 \%$ of the patients had a history of dual diagnoses. Patients with a history of depression or anxiety required a higher dose of buprenorphine during pregnancy $(\mathrm{p}=0.0217, \mathrm{p}=0.0165)$ 
compared to those without a history of mood disorder. There was no significant difference in the doses in patients with post-traumatic stress disorder versus controls. In those with a diagnosis of mood disorder, there was no difference in buprenorphine dose between women on medication versus those not on medication for depression, anxiety, and Post Traumatic Stress Disorder. There was no statistical difference between patients with or without mood disorder and the development of neonatal abstinence syndrome. For those that developed neonatal abstinence syndrome, infants whose mothers had anxiety or post-traumatic stress disorder required 2-6 extra days of morphine treatment compared to those infants of mothers without mood disorder $(\mathrm{p}=0.0088, \mathrm{p}=0.0291)$, no difference seen for depression or a combination of mood disorders. Development of neonatal abstinence syndrome or length of treatment did not vary if the mother was on medication for treatment of her mood disorder.

Conclusion: Pregnant women with a mood disorder require higher doses of buprenorphine compared to patients without a mood disorder. In women with mood disorders, there was no difference in buprenorphine dose in women treated with medication compared to those not taking medication for mood disorders. While, there was no difference in the incidence of neonatal abstinence syndrome in infants whose mothers also had a mood disorder, infants born of women with anxiety or post-traumatic stress disorder had longer stays at the Neonatal Intensive Care Unit as they needed 2-6 extra days of morphine treatment. These findings may help guide provider counseling of these women in discussion of post-delivery expectations.

\section{Introduction}

Opioid use disorder (OUD) is defined by The Diagnostic and Statistical Manual of Mental Disorder, Fifth Edition (DSM-V), as a pattern characterized by tolerance, craving, inability to control use, and continued use of opioids despite adverse consequences. ${ }^{1,2}$ This disorder affects all races, socioeconomic backgrounds, ethnicities, and geographic regions across the United States. ${ }^{3}$ Unfortunately, OUD has increased over the past decade, including an increased use in pregnancy of over $120 \%$ from 1998 to $2011 .^{2,3}$

The increase in use and misuse can be attributed to the opioids' mechanisms, which include diminishing the intensity of pain signals through opioid receptors, and causing a feeling of euphoria. ${ }^{1,4}$ Patients may become physically dependent with regular, long-term use; and manifest with a variety of withdrawal symptoms. Current pharmacotherapy treatment options for OUD rely on medications, like methadone and buprenorphine, that act upon the mu opioid receptors and prevent withdrawal symptoms. ${ }^{1}$ Methadone is a full opioid agonist with a long half-life that is dispensed on a daily basis through a treatment program. ${ }^{1,45}$ Unlike methadone, buprenorphine is a partial opioid agonist that makes overdose less likely, allowing it to be prescribed and administered at home..$^{1-5}$

Treatment of OUD in pregnancy can employ these opioid agonists and is geared towards a multi-disciplinary approach to include appropriate pregnancy care and fetal monitoring to decrease risk of pregnancy morbidities. ${ }^{1,3,4}$ Pregnant patients with OUD that do not seek medical assistance, have been shown to have decreased or lack of prenatal care; and increased risk of fetal growth restriction, placental abruption, fetal demise, preterm labor, neonatal abstinence syndrome, and meconium at the time of rupture of membranes.

Neonatal abstinence syndrome (NAS) is a drug withdrawal syndrome seen in $30-80 \%$ of pregnancies complicated by OUD, antidepressants, or opioid agonist therapies. ${ }^{1}$ With the increased used of opioids, the incidence of NAS increased from 1.5 to 6.0 cases per 1,000 hospital births from 1999 to 2013; representing a cost of $\$ 1.5$ billion. ${ }^{1}$ Neonates experience gastrointestinal and nervous system disturbances within 12 hours to 14 days of life; leading to irritability, high-pitched cry, and poor sleep. 'Studies have shown that symptoms do not depend on maternal dosage of methadone; but can vary with split methadone dosing or with the use of buprenorphine. ${ }^{9}$ Breastfeeding has also been associated decreased severity of NAS symptoms, less need for pharmacotherapy, and a shorter hospital stays. ${ }^{1}$

Although the severity of NAS symptoms has not yet been shown to be affected by maternal mental disease, studies have shown a positive correlation between maternal mental health disorders and the incidence of NAS. ${ }^{10}$ Faherty et al, demonstrated a $26 \%$ prevalence of depression in mothers of infants with NAS compared to infants of mothers without any mood disorder. ${ }^{11}$ Mothers with OUD and infants with NAS also had double the risk of depression compared to controls; as well as, 2.2 times the risk of bipolar disor- 
der, and 4.6 times the risk of schizophrenia. ${ }^{11}$ Other studies have also showed that mothers of infants with NAS had higher chance of suffering from anxiety and postpartum depression, with rates of $27 \%$ and $7 \%$ respectively, compared to mothers of infants without NAS. ${ }^{10}$

In addition to these pregnancy complications, women with OUD are also at increased risk of abusing non-opioid drugs and psychiatric disorders. ${ }^{1,6}$ Watkins et al found that approximately fifty percent of non-pregnant patients entering a substance abuse program screened positive for a co-occurring psychiatric illness with depression and anxiety being the most common. ${ }^{7}$ In those treated with methadone with either a mood disorder or anxiety disorder, patients were more likely to have a positive urine drug screen (UDS) and less chances of success with attempts of treatment for OUD in pregnancy. ${ }^{8}$

The purpose of this study is to compare the differences in buprenorphine doses needed to treat opioid use disorder in pregnant women with and without mood disorders; and to compare the development of neonatal abstinence syndrome in infants delivered to mothers treated with buprenorphine in patients with history of mood disorders versus those without mood disorder.

\section{Methods}

This a retrospective cohort study of pregnant women treated with buprenorphine for OUD through an outpatient OUD program initiated in March 2015 at Indiana University under the division of Maternal-Fetal Medicine. IRB approval was obtained for this study. Women were included in the study if they had at least one outpatient visit with our center during pregnancy during which they were prescribed buprenorphine and delivered within the Indiana University Health system. Exclusion criteria included patients that: 1) delivered outside of our hospital system and delivery information was unavailable; 2) had a miscarriage or fetal demise; 3) were undelivered by time of data collection; or 4) were non-compliant with an outpatient visit. After obtaining IRB approval, electronic health records were reviewed for maternal demographics, maternal medical history and prescription use, including dose at initiation and delivery, and delivery data. Upon initiation of buprenorphine, patients' social and medical history was recorded including history of current and prior illicit drug use, history of psychiatric disorder, prior psychiatric treatments or hospitalizations, and family history of psychiatric illness and drug use.

Analyses were performed to determine if there was a significant association between the maximum buprenorphine dose used in our clinic (24mg) and outcomes of interest. Maximum dose was determined by taking the maximum out of the four dosing times or periods (first, second, and third trimester and delivery). Student's t-tests and Analysis of Variance models were performed when comparing dose across levels of categorical variables for two and more than two levels, respectively. Chi-Square tests were used when testing for homogeneity between levels of categorical variables. Correlation analyses were performed when comparing continuous variables to dose. All analytic assumptions were verified. When data were skewed, non-parametric tests were performed, as data transformations were unable to correct the skewness, with Wilcoxon-Kruskal-Wallis rank-sum and Spearman correlation analyses being performed. Fisher's Exact tests were used to verify the results of Chi-Square tests when expected cell counts were small. All analyses were performed using SAS v9.4 (SAS Institute, Cary, NC).

\section{Results}

Four-hundred and nine pregnancies were treated through our clinic from March 2015 to December 2018. One-hundred and forty-three were excluded due to one of the exclusion criteria. Of the remaining 266 pregnancies, 171 were noted to have either a history of mood disorder or were actively receiving medical treatment for mood disorders. The three mood disorders reviewed included depression $(n=148)$, anxiety $(n=130)$ and post-traumatic stress disorder (PTSD, $n=19$ ). Over forty percent of the patients had diagnosis of more than one mood disorder.

There was no significance differences in maternal age, marital status, education level, or parity of subjects with or without a mood disorder (Table 1). The average age of first drug use was approximately 20 years old with no difference between the groups. While the first substance used was more likely to be opioid pills in both groups $(51.6 \%$ with no mood disorders vs. $49.4 \%$ with); those without a mood disorder were more likely to have used heroin and those with mood disorder were more likely to have used benzodiazepines $(\mathrm{p}=0.0046)$. There was no difference in tobacco use among the groups. 


\begin{tabular}{|c|c|c|c|}
\hline & $\begin{array}{l}\text { No mood disorders } \\
\qquad(\mathrm{n}=95)\end{array}$ & $\begin{array}{c}\text { One or more mood } \\
\text { disorders } \\
(n=171)\end{array}$ & P-value \\
\hline Maternal Age & $28.81(4.82)$ & $28.64(4.71)$ & .7833 \\
\hline \multicolumn{4}{|l|}{ Race } \\
\hline White & $91(95.8)$ & $171(100)$ & $.0156^{*}$ \\
\hline Black & $4(4.2)$ & $0(0)$ & \\
\hline \multicolumn{4}{|l|}{ Education } \\
\hline$<$ High School & $5(5.4)$ & $9(5.4)$ & .4519 \\
\hline High School & $59(64.1)$ & $119(71.3)$ & \\
\hline$>$ High School & $28(30.4)$ & $39(23.4)$ & \\
\hline Employed & $32(33.7)$ & $45(26.3)$ & .2042 \\
\hline \multicolumn{4}{|l|}{ Marital Status } \\
\hline Single & $69(72.6)$ & $111(64.9)$ & .5811 \\
\hline Married & $18(19.0)$ & $45(26.3)$ & \\
\hline Separated & $4(4.2)$ & $8(4.7)$ & \\
\hline Divorced & $4(4.2)$ & $7(4.1)$ & \\
\hline Nulliparous & $20(21.1)$ & $29(17.0)$ & .4092 \\
\hline Tobacco use (yes) & $83(87.4)$ & $149(87.1)$ & .9563 \\
\hline Prior treatment attempts (yes) & $49(62.0)$ & $115(74.7)$ & $.0453 *$ \\
\hline Gestational age at initiation & $20.01(8.79)$ & $18.92(7.88)$ & .3005 \\
\hline Age at First Use & $20.32(6.14)$ & $19.81(5.21)$ & .4927 \\
\hline \multicolumn{4}{|l|}{ First Substance Used } \\
\hline Opioid Pills & $49(51.6)$ & $84(49.4)$ & $.0046^{*}$ \\
\hline Heroin & $23(24.2)$ & $22(12.9)$ & \\
\hline THC & $20(21.1)$ & $45(26.5)$ & \\
\hline $\mathrm{BDZ}$ & $1(1.1)$ & $17(10.0)$ & \\
\hline Methamphetamine & $1(1.1)$ & $2(1.2)$ & \\
\hline Cocaine & $1(1.1)$ & $0(0)$ & \\
\hline \multicolumn{4}{|l|}{ Drug of Choice } \\
\hline Opioid Pills & $39(41.1)$ & $70(41.2)$ & .5502 \\
\hline Heroin & $55(57.9)$ & $100(58.8)$ & \\
\hline Methamphetamine & $1(1.1)$ & $0(0)$ & \\
\hline
\end{tabular}

(Table 1)

Values are means (standard deviations) for continuous variables and frequencies (percentages) for categorical variables. P-values come from Student's t-tests and Chi-Square tests, respectively, with Fisher's Exact tests being used instead of Chi-Square in cases where cell counts were low. Frequencies may not add to 166 due to missing data. 
Both groups initiated treatment of OUD with buprenorphine in pregnancy during the mid-second trimester, but patients with mood disorder were more likely to have had prior treatment attempts in the past ( $74.7 \%$ vs. $62.0 \%$; $\mathrm{p}=0.0453)$. When compared to patients without mood disorders, those with depression or anxiety required a significantly higher maximum dose of buprenorphine (median doses for both disorders $16 \mathrm{mg}$ vs. $20 \mathrm{mg}$; $\mathrm{p}=0.2017$ and $\mathrm{p}=0.0165$, respectively). Maximum dose of buprenorphine used in our clinic for treatment of OUD in pregnancy is $24 \mathrm{mg}$, at which point patients are offered treatment with methadone instead buprenorphine for further management of OUD. There was no significant difference in the doses of buprenorphine for those with PTSD alone (Figure 1).

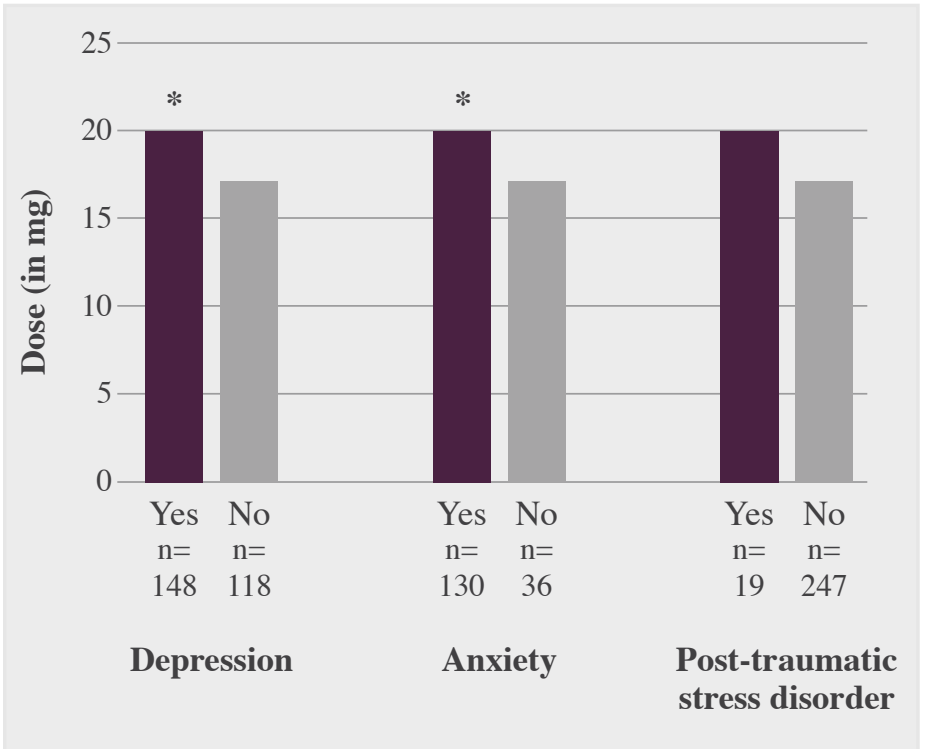

(Figure 1)

Maximum buprenorphine dose (in mg) required in patients with $v$ s. without mood disorder $(n=266)$

Non-parametric rank-sum analyses performed by Wilcoxon rank-sum test $* p<0.05$

The dose of buprenorphine was then compared to those patients with mood disorder receiving active medication treatment of their mood disorder versus those not receiving treatment. Patients treated for depression, anxiety, or PTSD had no difference in their maximum required dose of buprenorphine compared to those not actively prescribed medication. There was also no difference in buprenorphine dose those with multiple mood disorders (Figure 2).

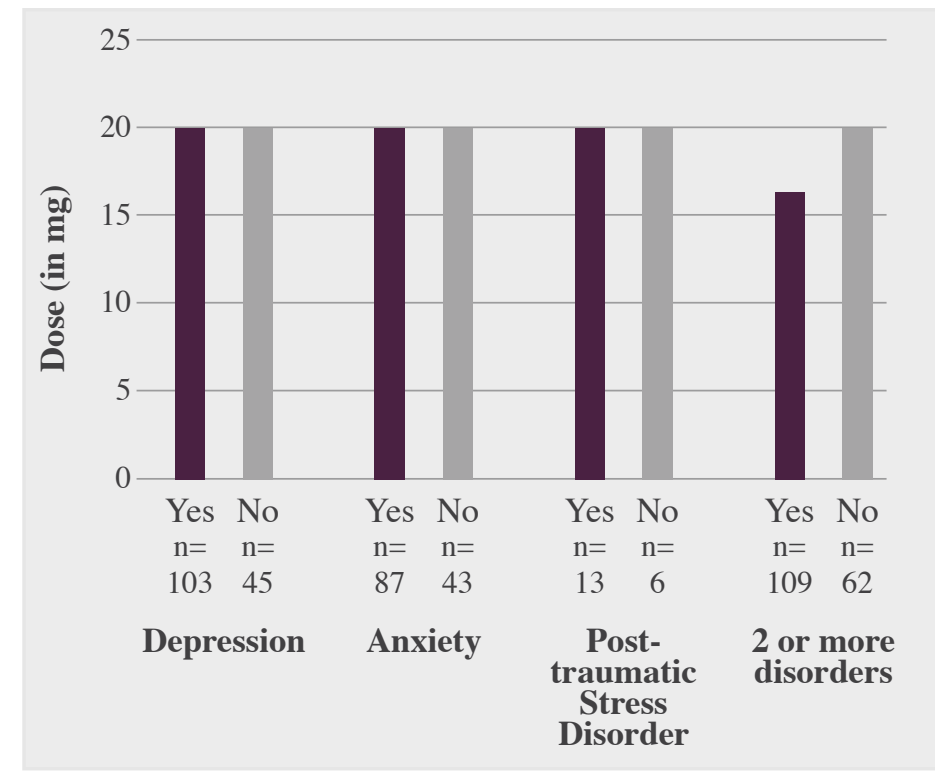

(Figure 2) Maximum buprenorphine dose (in mg) required
in patients with treated mood disorder vs. those without treatment

Non-parametric rank-sum analyses performed by Wilcoxon rank-sum test

Yes=receiving treatment for mood disorder No=no treatment for mood disorder

Results also showed that there was no difference in the development of NAS in infants of mothers with and without mood disorders (Table 2). In cases of depression, the difference of NAS was $56.5 \%$ vs $43.5 \%(\mathrm{p}=0.65)$; meanwhile for anxiety, it was $52.2 \%$ vs $47.8 \%(\mathrm{p}=0.22)$, and $6.5 \%$ vs 93.5\% for PTSD ( $\mathrm{p}=0.744)$. Even for cases of combined mood disorders, there was no difference in the incidence of NAS with $66.7 \%$ vs $33.3 \%$ ( $\mathrm{p}=0.3053$ ). These differences in the incidence of NAS were not affected by the use of psychiatric treatment (depression $\mathrm{p}=0.66$; anxiety $\mathrm{p}=0.50$ PTSD $\mathrm{p}=0.59$, Table 4).

If NAS did develop, the length of hospital stay varied depending on the mood disorder (Table 3). Infants of mothers with anxiety and PTSD had a longer duration of NAS related symptoms, $6-51$ vs $2-42$ days ( $\mathrm{p}=0.0088$ ) and $10-25$ vs $2-52$ days $(\mathrm{p}=0.0291)$ respectively; compared with combined disorders and depression which had a NAS symptoms duration of 2-51 days ( $\mathrm{p}=0.38$ and 0.16 respectively). No difference in length of stay was seen in infants of mothers being treated for their mood disorders (Table 5). There were also no statistically significant differences between birth weight and 


\begin{tabular}{|c|c|c|c|}
\hline & NAS (no) & NAS (yes) & $\begin{array}{c}\text { P-value } \\
\text { chi-square } \\
\text { test }\end{array}$ \\
\hline Depression & $55(46.2)$ & $60(43.5)$ & .6596 \\
\hline No & $64(53.8)$ & $78(56.5)$ & \\
\hline Yes & & & \\
\hline Anxiety & $66(55.5)$ & $66(47.8)$ & .2220 \\
\hline No & $53(44.5)$ & $72(52.2)$ & \\
\hline Yes & $110(92.4)$ & $129(93.5)$ & .7443 \\
\hline PTSD & $9(7.6)$ & $9(6.5)$ & \\
\hline No & $72(60.5)$ & $92(66.7)$ & \\
\hline Yes & & & \\
\hline $\begin{array}{l}\text { Any combination } \\
\text { one of those three) }\end{array}$ & $\begin{array}{l} \\
\text { No }\end{array}$ & & \\
\hline Yes & & & \\
\hline
\end{tabular}

(Table 2)

Differences in NAS with mood disorders. Values are frequencies (percentages).

incidence of NAS when comparing races. There was also no difference in NAS development in breastfed infants among those patients with mood disorders.

\section{Discussion}

While the safety and efficacy of buprenorphine for the treatment of OUD in pregnancy has been well established, there is little information available on the effect of underlying mood disorders on treatment dosage. Prior studies in both pregnant and non-pregnant patients have noted that a portion of these patients have additional underlying psychiatric disease that may impact treatment success. However, there are no prior studies to our knowledge that have evaluated buprenorphine dose requirements in pregnant women with OUD and mood disorders.

Our results showed that more than half of our patients

\begin{tabular}{|c|c|c|}
\hline & NAS duration & $\begin{array}{c}\text { P-value from } \\
\text { Wilcoxon test }\end{array}$ \\
\hline Depression & $12(5-42)$ & .3887 \\
\hline No & $13(2-51)$ & \\
\hline Yes & $11(2-42)$ & $.0088^{*}$ \\
\hline Anxiety & $13(6-51)$ & \\
\hline No & $12(2-51)$ & $.0291^{*}$ \\
\hline Yes & $18(10-25)$ & \\
\hline PTSD & & \\
\hline No & $11(5-42)$ & .1691 \\
\hline Yes & $13(2-51)$ & \\
\hline $\begin{array}{l}\text { Any combination } \\
\text { (where YES for at least } \\
\text { one of those three) }\end{array}$ & & \\
\hline No & & \\
\hline \begin{tabular}{l} 
Yes \\
\hline
\end{tabular}
\end{tabular}

(Table 3)

Differences in NAS DURATION with mood disorders. Values are medians (ranges).

$* p<0.05$

treated with buprenorphine for OUD also have a diagnosed mood disorder; with up to $40 \%$ having 2 or more mood disorders. This correlates with findings of the general population which show approximately $32 \%$ of patients have concurrent opioid use and mood disorders. ${ }^{12}$ In our population, mood disorder diagnosis did not significantly vary by age, marital status, education level, or parity of women. The existence of a mood disorder did influence dosage requirements, as patients with depression or anxiety required a higher maximum dose of buprenorphine. The increase of buprenorphine dosage in pregnancy without accounting for mood disorders is well known. Caritis et al concluded that pregnant patients required more frequent dosing intervals to sustain plasma concentrations of buprenorphine of more than $1 \mathrm{ng} / \mathrm{mL}$ to prevent withdrawal symptoms and improve adherence. ${ }^{13}$ However, knowing that there is an additional possible increase in dosing needed with mood disorders based on this study, can potentially help guide the pre-pregnancy and pregnancy counseling of these women. 


\begin{tabular}{|c|c|c|c|}
\hline & NAS (no) & NAS (yes) & $\begin{array}{c}\text { P-value } \\
\text { chi-square } \\
\text { test }\end{array}$ \\
\hline Depression (YES only) & & & \\
\hline Meds No & $21(32.8)$ & $23(29.5)$ & .6698 \\
\hline Meds Yes & $43(67.2)$ & $55(70.5)$ & \\
\hline Anxiety (Yes only) & & & \\
\hline Meds No & $20(37.7)$ & $23(31.9)$ & .5006 \\
\hline Meds Yes & $33(62.3)$ & $49(68.1)$ & \\
\hline PTSD (Yes only) & $3(33.3)$ & $2(22.2)$ & .5987 \\
\hline Meds No & $6(66.7)$ & $7(77.8)$ & \\
\hline $\begin{array}{l}\text { Meds Yes } \\
\text { (where YES for at least } \\
\text { one of those three) }\end{array}$ & $28(38.9)$ & $32(34.8)$ & .5880 \\
\hline $\begin{array}{l}\text { Meds No } \\
\text { Meds Yes }\end{array}$ & $44(61.1)$ & $60(65.2)$ & \\
\hline
\end{tabular}

(Table 4)

Differences in NAS with treatment for those with mood disorders. Values are frequencies (percentages).

$$
* p<0.05
$$

The results also provide data to develop better plans of care for patients during their pregnancy; and discuss realistic pregnancy and postpartum expectations with patients. For example, although there was no increased risk of NAS development in infants of mothers with mood disorders, there was an increased length of hospital stay for infants of mothers being treated for anxiety and PTSD. Therefore, the prenatal care of these patients should include discussion of possible neonatal expectations and side effects after delivery.

One of the strengths of this study is that our treatment group in pregnancy represents a large group of patients on buprenorphine in a single center. We believe that having an active treatment center for patients with OUD in pregnancy allows for improvements in pregnancy care through a single office compared to patients requiring treatment by both an OUD center and obstetrics.

However, the study is limited by its retrospective nature. We

\begin{tabular}{|c|c|c|}
\hline & NAS duration & $\begin{array}{c}\text { P-value from } \\
\text { Wilcoxon test }\end{array}$ \\
\hline Depression (YES only) & & \\
\hline Meds No & $11.5(2-51)$ & .1247 \\
\hline Meds Yes & $13.5(6-41)$ & \\
\hline Anxiety (Yes only) & & \\
\hline Meds No & $13(7-51)$ & .8092 \\
\hline Meds Yes & $14(6-41)$ & \\
\hline PTSD (Yes only) & & .7472 \\
\hline Meds No & $16.5(13-20)$ & \\
\hline Meds Yes & $18(10-25)$ & \\
\hline $\begin{array}{l}\text { Any combination } \\
\text { (where YES for at least } \\
\text { one of those three) }\end{array}$ & & \\
\hline Meds No & $12(2-51)$ & .3650 \\
\hline \begin{tabular}{c} 
Meds Yes \\
\hline
\end{tabular} & $13(6-41)$ & \\
\hline
\end{tabular}

(Table 5)

Differences in NAS DURATION with treatment for those with mood disorders.Values are medians (ranges).

$$
* p<0.05
$$

were limited by information available in the medical records over a 3-year period. Some of the patients were included based on a self-reported history of psychiatric illnesses and not confirmed via prior validated testing in patients not on current medication for their mood disorder. In addition, some of the sample sizes for certain mood disorders, like PTSD, were small.

\section{Conclusion}

The risk of NAS in mothers with OUD has been studied extensively but there is limited data on how mood disorders affect the risk of NAS in this population. This study provides data that can help guide the providers for proper counseling of these women in discussion of their pregnancy and post-delivery expectations. Results from this study show that a difference in dosage exists, and that patients with 


\section{Acknowledgments}

We would like to thank the ancillary staff of the Indiana University School of Medicine Maternal Fetal Medicine office who assisted in collecting patient lists from the time of initiation of this program for medical record review.

\section{References}

1. Gynecologists, A.C.o.O.a., Opioid Use and Opioid Use in Pregnancy. Committee Opinion No 711. Obstetrics and Gynecology, 2017. 130: p. e81-94.

2. Krans, E.E. and S.W. Patrick, Opioid Use Disorder in Pregnancy: Health Policy and Practice in the Midst of an Epidemic. Obstetrics and Gynecology, 2016. 128(1): p. 4-10.

3. Haycraft, A.L., Pregnancy and the Opioid Epidemic. Journal of Psychosocial Nursing and Mental Health Services, 2018. 56(3): p. 19-23.

4. Stanhope, T.J., L.A. Gill, and C. Rose, Chronic opioid use during pregnancy: maternal and fetal implications. Clinics in Perinatology, 2013. 40(3): p. 337-50.

5. Meyer, M. and J. Phillips, Caring for pregnant opioid abusers in Vermont: A potential model for non-urban areas. Preventive Medicine, 2015. 80: p. 18-22.

6. Holbrook, A. and K. Kaltenbach, Co-occurring psychiatric symptoms in opioid-dependent women: the prevalence of antenatal and postnatal depression. American Journal of Drug and Alcohol Abuse, 2012. 38(6): p. 575-9.

7. Watkins, K.E., et al., Prevalence and characteristics of clients with co-occurring disorders in outpatient substance abuse treatment. American Journal of Drug and Alcohol Abuse, 2004. 30(4): p. 749-64.

8. Fitzsimons, H.E., et al., Mood disorders affect drug treatment success of drug-dependent pregnant women. Journal of Substance Abuse Treatment, 2007. 32(1): p. 19-25.

9. Stover, M., et al., Opioids in Pregnancy and Neonatal Abstinence Syndrome. Seminars in Perinatology, 2015. 39(7): 561-565
10. Pediatric Academic Societies. "Mothers of children born with NAS are more likely to experience mental health problems." ScienceDaily, 5 May 2018. www.sciencedaily.com/ releases/2018/05/180505091609.htm.

11. Faherty, L., et al., Mental Health of Mothers of Infants with Neonatal Abstinence Syndrome and Prenatal Opioid Exposure. Maternal and Child Health Journal, 2018. 22(6): 841-848

12. Quello, S, et al. Mood Disorders and Substance Use Disorder: A Complex Comorbidity. Science and Practice Perspectives. Dec 2005. 3(1): 13-21.

13. Caritis, S, et al., An evidence-based recommendation to increase the dosing frequency of buprenorphine during pregnancy. American Journal of Obstetrics and Gynecology, 2017. 217:459, e1-6. 